\title{
Research on Rectal Tumor Identification Method by Convolutional Neural Network Based on Multi-Feature Fusion
}

\author{
Zhuang Liang(1), Jiansheng $\mathbf{W u}^{\left(1^{*}\right)}$ \\ (1) School of Computer Science and Software Engineering, University of Science and Technology Liaoning, Anshan 114044, \\ CHINA \\ e-mail: ssewu@163.com
}

\section{SUMMARY}

Aiming at the obscure features of tumors in rectal CT images and their complexity, this paper proposes a multi-feature fusion-based convolutional neural network rectal tumor recognition method and uses it to model rectal tumors for classification experiments. This method extracts the convolutional features from rectal CT images using Alexnet, VGG16, ResNet, and DenseNet, respectively. At the same time, local features such as histogram of oriented gradient, local binary pattern, and HU moment invariants are extracted from this image. The above local features are fused linearly with the convolutional features. Then we put the new fused features into the fully connected layer for image classification. The experimental results finally reached the accuracy rates of $92.6 \%, 93.1 \%, 91.7 \%$, and $91.1 \%$, respectively. Comparative experiments show that this method improves the accuracy of rectal tumor recognition.

KEYWORDS: Rectal CT images; convolutional neural networks; multi-features; image recognition; data fusion.

\section{INTRODUCTION}

As one of the most common malignant tumors, colorectal cancer has received widespread attention in China. Of the 1.36 million cases of colorectal cancer diagnosed worldwide in 2012, the number of new cases in China reached 253,000, accounting for $18.6 \%$ of new cases worldwide. Therefore, it is vital to carry out active cancer prevention and treatment countermeasures to reduce colorectal cancer incidence and mortality rate in China. With the improvement of social and economic standards, people's dietary structure has changed dramatically. As a result, the incidence of colon cancer is increasing year by year. However, the diagnosis and management of rectal cancer remain challenging. Due to its anatomical structure, rectal cancer is delicate to operate on, has a high complication rate and local recurrence rate. This disease seriously impacts the quality of life of patients [1]. Therefore, 
identifying rectal cancer has become an essential part of the subsequent diagnosis and treatment.

Nowadays, medical experts can detect rectal cancer by observing CT images, but it relies on subjective factors such as the knowledge and experience of clinical experts. With the constant development of CT image scanning technology, doctors are continuously looking for suspicious tumor areas inspecting a large number of rectal images. This repetitive procedure inevitably produces visual fatigue and eventually leads to misdiagnosis.

With the advancement in information technology, computer-aided diagnosis has been adopted in various fields. Many research methods based on computer-aided diagnosis have been proposed for image classification in the medical field. These methods are mainly divided into traditional image feature extraction methods and deep learning methods. Because the traditional image feature extraction methods are not commonly used in the classification of rectal tumors, the information can be acquired from the extraction methods used in other medical diseases. Mahmoud et al. [2] used the discrete wavelet transform to extract features from segmented breast tumor images and then used a combination of principal component analysis and linear discriminant analysis to reduce the dimensionality of the extracted features. The statistical classifier was applied to classify benign and malignant tumors from the images. Huang et al. [3] used a Gaussian Markov random field and a local banalization algorithm to extract the texture features from the prostate tumor images and put them into the SVM classifier. Ayadi et al. [4] first normalized brain tumor images, then from images extracted speeded up robust (SURF) features and histogram of oriented gradient (HOG) features and generated discriminative feature sets, and finally put those sets into a support vector machine. Aarthy et al. [5] applied gray-level co-occurrence matrix (GLCM) for feature extraction from breast tumor images, then developed an effective nonlinear heat conduction identification method and finally classified the input feature information with support vector machines. Usman et al. [6] first extracted features such as intensity, intensity difference, local neighborhood, and wavelet texture from pre-processed brain tumor images and input them into a random forest classifier for image classification.

With the introduction of deep learning, its representative convolutional neural network has been widely used in the medical field. Yao et al. [7] proposed a transfer learning based VGG network for colorectal tumor image recognition. In the method, ImageNet pre-training network parameters are transferred to the VGG network with training colorectal data to acquire good initial network performance. Nguyen et al. [8] performed high-throughput TMA kernel extraction and color enhancement on colorectal tumor images and then placed the processed images into a VGG network for image recognition. Sahadev et al. [9] present a robust architecture for endoscopic image classification using an efficient dilation in Convolutional Neural Network (CNNs). Natinai et al. [10] used Xception network model and swish function for colorectal polyps` image recognition. Michał et al. [11] proposed a two-stage resampling method for convolutional neural network training in the imbalanced colorectal cancer image classification.

The convolutional neural network extracts the deep features of the image from a large number of samples, and the traditional machine learning method extracts the local features of the image. Therefore, this study developed a rectal tumor recognition method based on multifeature fusion, which combines a convolutional neural network with traditional image feature extraction methods. 


\section{METHODOLOGY}

In this study, histograms of oriented gradient, local binary pattern (LBP), and HU moment invariants were fused with Alexnet, VGG, ResNet, and DenseNet convolutional neural networks, respectively, to achieve rectal tumor recognition.

\subsection{TRADITIONAL IMAGE FEATURE EXTRACTION}

\subsubsection{HOG}

HOG, proposed by Navneet Dalal [12] in 2005, is a feature extraction method performed by computing a histogram of gradient directions in local regions of an image. Firstly, the CT image is greyed out. Secondly, the CT image is gamma normalized to reduce the effect of the shadow of the CT image and illumination variation. Then the horizontal and vertical gradients of the CT image are calculated using the Sobel operator, respectively, and the specific calculation formula is as follows:

$$
\begin{gathered}
G_{x}(x, y)=h(x+1, y)-h(x-1, y) \\
G_{y}(x, y)=h(x, y+1)-h(x, y-1) \\
G(x, y)=\sqrt{G_{X}(x, y)^{2}+G_{y}(x, y)^{2}} \\
\alpha(x, y)=\tan ^{-1}\left(\frac{G_{y}(x, y)}{G_{x}(x, y)}\right)
\end{gathered}
$$

where $G_{x}(x, y), G_{y}(x, y)$ represent the horizontal and vertical gradients of the CT image at pixel point $(x, y)$, respectively, and $h(x, y)$ represents the pixel value of the CT image at pixel point $(x, y) . \mathrm{G}(x, y)$ represents the gradient magnitude, $\alpha(x, y)$ represents the gradient direction of the CT image at pixel point $(x, y)$.

Finally, the CT image is divided into several local cells of $8 \times 8$ pixels, and all $2 \times 2$ local cells form an interval block. The gradient direction $\alpha(x, y)$ is then converted to 9 directional blocks (bins) in the interval $\left[0-180^{\circ}\right]$, i.e., every $20^{\circ}$ is a directional block, and the function is to count the gradient histogram of different local cells. Suppose the sample size of the CT image is $48 \times 48$, and 8 pixels are used as sliding windows for extracting image features, thus there are 5 sliding windows horizontally and 5 vertically. Finally, the gradient histograms of all blocks are connected to form the HOG features of the CT image, i.e., the dimensions of the HOG features extracted from each CT image is $2 \times 2 \times 9 \times 5 \times 5=900$.

\subsubsection{LBP}

LBP, proposed by Timo Ojala [13] in 1994, is applied to extract local texture features from images. First, the CT image is greyed out. Then a $3 \times 3$ sliding window with a step size of 1 is defined to extract the image features. The center pixel value of the window is the standard, compared with the adjacent 8 pixel values. If the adjacent pixel value is greater than the center pixel value, the position is set to 1 , otherwise set to 0 . Finally, it is arranged in the adjacent order to obtain an 8 - bit binary number and this binary number is converted to a decimal number to get the LBP value of the center region pixel. The specific calculation formula is as follows:

$$
\operatorname{LBP}\left(x_{c}, y_{c}\right)=\sum_{p=0}^{p-1} 2^{p} \cdot S\left(i_{p}-i_{c}\right)
$$


where $\left(x_{c}, y_{c}\right)$ represents the center pixel point, $\mathrm{i}_{c}$ is the center pixel value, and $i_{p}$ is the pixel value of the neighboring pixels. If $\mathrm{x}$ is greater than or equal to $0, S(x)$ is set to 1 , otherwise, $S(\mathrm{x})$ is set to 0 .

The final dimensionality of the LBP features extracted from each CT image is $48 \times 48=2304$.

\subsubsection{HU MOMENT INVARIANTS}

HU moment invariants [14] extract geometric features of images with invariant properties such as rotation, translation, etc. First, the CT image is greyed out. Then set the grayscale distribution of the CT image as $f(x, y)$, and its moments of $(p+q)$ order $m_{p q}$ and normalized moments $\mu_{p q}$ are obtained as follows:

$$
m_{p q}=\sum_{x=1}^{M} \sum_{y=1}^{N} x^{p} \cdot y^{p} \cdot f(x, y)
$$

where $M, N$ represent respectively the length and width of this CT image, and $\forall p, q \in\{1, \ldots, n\}$ with the center of gravity of the moments $\left(x_{0}, y_{0}\right)$ :

$$
x_{0}=\frac{m_{10}}{m_{00}}, y_{0}=\frac{m_{01}}{m_{00}}
$$

The normalized central moment $v_{p q}$ is obtained from the normalized moment $\mu_{p q}$ :

$$
v_{p q}=\frac{\mu_{p q}}{\mu_{00}^{\beta}}
$$

where $\beta=(p+q+2) / 2, p+q \in\{2, \ldots, n\}$.

Finally, seven Hu invariant moments were constructed according to the second and third order normalized central moments used as the invariant moment features of the CT image.

\subsection{DEEP CONVOLUTIONAL FEATURE EXTRACTION}

Convolutional neural networks have been widely used in the medical field with good results. Convolutional neural networks can extract deep convolutional features of images, which have deeper image information compared with the features extracted by traditional machine learning. Therefore, four deep learning networks, such as Alexnet, VGG16, ResNet, and DenseNet, are selected to extract deep convolutional features of CT images in this study.

\subsubsection{ALEXNET}

In 2012, Alex Krizhevsky [15] proposed Alexnet in the ImageNet competition and won. The Alexnet network structure contains five convolutional layers and three fully connected layers, each followed by a maximum pooling layer.

The advantages of this network model are as follow:

- The use of a nonlinear activation function, Relu, which reduces the computational effort of the model and prevents the gradient disappearance and overfitting of the model.

- The local corresponding normalization layer (LBN) is added, whose role is to imitate the human brain neural units by creating a competitive mechanism to improve the accuracy of recognition and effectively increase the generalization ability of the network model. 


\subsubsection{VGG16}

VGG16[16] was proposed by the Oxford University Computer Vision Group (Visual Geometry Group) at the ILSVRC 2014 competition, where it achieved second place. It makes some improvements based on the Alexnet network: The size of the convolution kernel of the model was uniformly modified to the size of $3 \times 3$, and the size of the maximum pooling layer was also modified to the size of $2 \times 2$, which greatly reduced the number of calculated parameters. Secondly, the original 8-layer network structure is increased to 16 layers, which greatly increases the network's generalization ability.

\subsubsection{RESETNET}

With the increase of the number of network layers, the network has more parameters, and the deep structure means that the expression ability of the network is strong. However, due to the deepening of the number of network layers, the performance of the network reaches saturation, resulting in slow convergence of the network, poor classification effect, and other problems. This problem is also called the "degradation" problem of the network. To solve this problem, HE [17] et al. proposed a residual network structure in 2015. The network structure is designed to achieve a constant mapping between input and output. Compared with other neural networks, the residual network changes the learning objective, which is not to learn a complete output, but the difference between output and input, that is, the residual.

\subsubsection{DENSENET}

DenseNet [18] was proposed by Gao et al. in 2017. The network consists of a dense block and a transition layer. The purpose of a dense block is to closely link the output of all previous layers with the input of the current layer. The transition layer consists of the Batch Normalization layer, $1 \times 1$ convolutional layer, and $2 \times 2$ max-pooling layer. Its specific function is to reduce the number of channels in the image feature layer by using the $1 \times 1$ convolution layer and to reduce the distribution rate of image features by using the maximum pooling layer, which greatly reduces the number of network parameters and enhances the robustness of the network model. The thick block consists of several convolutional blocks. Let there be $\mathrm{M}$ convolutional blocks in this thick block, $\mathrm{x}_{0}$ is the input of the first convolutional block, $x_{m}$ is the output of the $M^{\text {th }}$ convolutional block, then $x_{m}$ can be expressed as:

$$
x_{m}=F_{m}\left(\left[x_{0}, x_{1}, \ldots, x_{m}\right]\right), \forall m=\{0, \ldots, M\}
$$

where the $F_{m}(-)$ function is represented as the nonlinear output after a convolutional block. [ $x_{0}$, $\left.x_{1}, \ldots, x_{m}\right]$ represents a linear merging of $x_{0}$ to $x_{m}$.

DenseNet has several advantages over previous network structures:

- Reduces the risk of gradient disappearance occurring.

- Reinforces the connection between different feature layers and makes more effective use of the feature information of different feature layers.

- Reduces the number of parameters of the network model and prevents the phenomenon of overfitting. 


\subsection{RECTAL TUMOR IDENTIFICATION BASED ON MULTI-FEATURE FUSION}

In this study, we propose a multi-feature fusion-based rectal tumor recognition method, in which the features extracted according to traditional machine learning 3211 dimensions are fused linearly with the deep convolutional features extracted from four deep learning networks, such as Alexnet, Vgg16, Resnet, DenseNet. Let there be the HOG feature vector, LBP feature vector, moment invariants of an image extracted feature vectors and deep convolutional feature vectors as $\left[a_{1}, a_{2}, \ldots, a_{q}\right],\left[b_{1}, b_{2}, \ldots, b_{w}\right],\left[c_{1}, c_{2}, \ldots, c_{e}\right],\left[d_{1}, d_{2}, \ldots, d_{r}\right]$, respectively, then the overall feature vectors after fusion are:

$$
L=\left[a_{1}, a_{2}, \ldots, a_{q}, b_{1}, b_{2}, \ldots, b_{w}, c_{1}, c_{2}, \ldots, c_{e}, d_{1}, d_{2}, \ldots, d_{r}\right]
$$

The fused features are fed into the fully connected layer for classification, and the final prediction is based on the softmax function, calculated as follows:

$$
\operatorname{softmax}\left(o_{i}\right)=\frac{e^{o^{i}}}{\sum_{j=1}^{n} e^{o^{j}}}
$$

where $O_{i}$ is the output value of the $i^{\text {th }}$ node, and $n$ represents the number of categories, here taken as 2 . The softmax function can be used to change the output value to a probability value between $(0,1)$, and the sum of the output values is 1 , which facilitates the classification calculation. The architecture of the network is as shown in Figure 1.

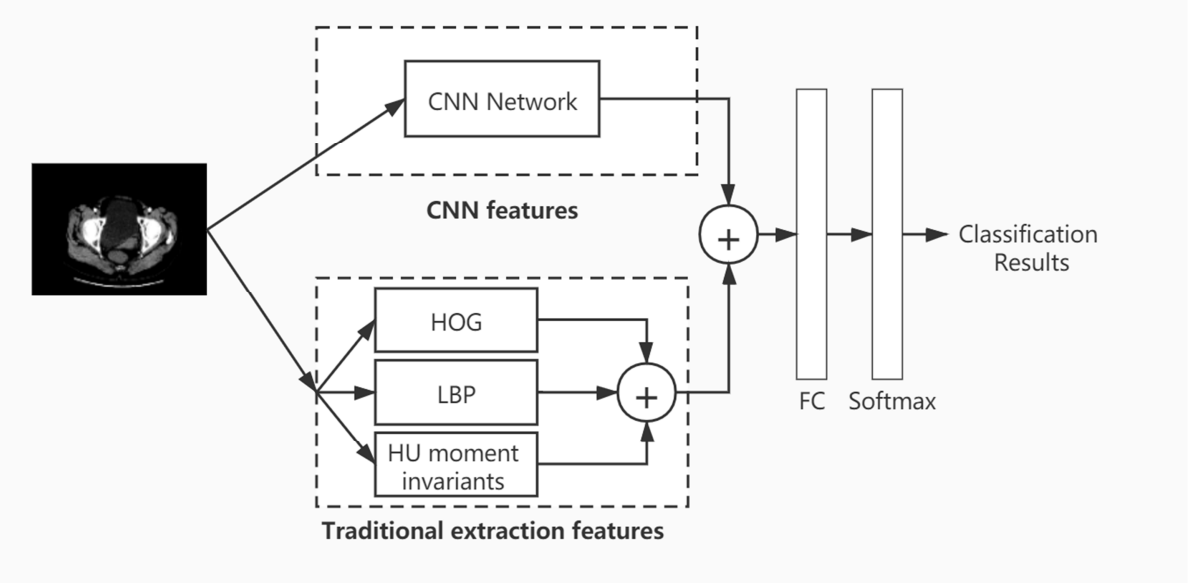

Fig. 1 The architecture of network for identification of rectal tumors by multi-feature fusion

\section{EXPERIMENTAL RESULTS AND ANALYSIS}

\subsection{EXPERIMENTAL ENVIRONMENT}

The experimental environment of this study is divided into hardware environment and software environment. The hardware environment comprises: CPU is INTEL i7-8750H with 2.2GHz, GPU is NVIDIA GeForce GTX 1070 with 8G video memory; and the software environment: the system is Window10, the deep learning framework is Tensorflow2.0, the Python version is 3.6. In this experiment, the number of the epoch is set to 50 , the optimizer is Adam, the learning rate is 0.0001 , and the loss function is categorical_crossentropy function. 


\subsection{EVALUATION INDEX}

To assess the effectiveness of the method of this study, the experimental evaluation indexes of this study were all quantitatively evaluated by the ACC coefficient. The index is calculated as follows:

$$
\text { Accuracy }=\frac{T N+T P}{T N+T P+F P+F N}
$$

Where TP refers to true positives, indicating the number of cases accurately identified as positive, TN to true negatives, the number of those accurately identified as negative, FP to false positives, the number of those incorrectly identified as positive, and FN to false negatives, the number of those incorrectly identified as negative.

\subsection{IMAGE DATASET}

\subsubsection{DATA PREPROCESSING}

The experimental dataset used in this study is from the $7^{\text {th }}$ Teddy Cup Data Mining Challenge B. The dataset is divided into two sets of CT images: arterial phase and venous phase. In this study, the CT images of the arterial phase were used, containing a total of 3029 images and the corresponding image masks. The CT image sample size of this data set is $512 \times 512$ grayscale images. To reduce the memory requirement of the graphics card, the images are normalized to $48 \times 48$ three-channel color images. The normalization process normalizes the image pixel values to between $[0,255]$. Then the image windowing process is performed to enhance the contrast of the CT image, whose effect is shown in Figure 2. Finally, the data category label is established according to the image mask graph. The specific method extracts the data matrix of the image mask graph and sums them up according to the matrix information. If the value is 0 , the image is negative, then the data category label is 0 ; otherwise, the image is positive, and the data category label is 1 .

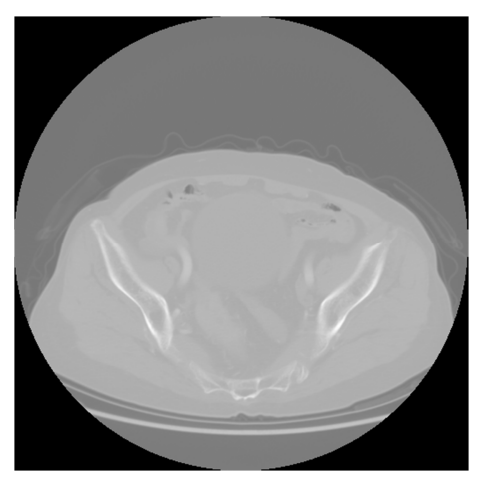

(a) Original Image

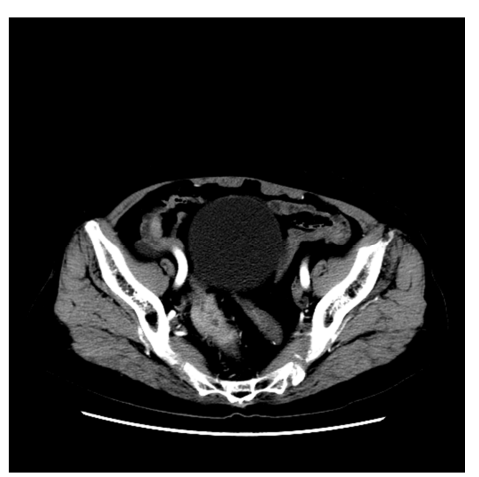

(b) Window processed image

Fig. 2 Window processing effect

\subsubsection{DATASET EXPANSION AND PARTITIONING}

Deep learning requires large datasets. The original dataset was expanded by flipping horizontally, rotating $45^{\circ}, 90^{\circ}$, and $180^{\circ}$ to make the dataset five times larger, with a total of 15,145 images. The sample data set was divided into training, validation, and test sets in the 
ratio of $6: 2: 2$. The training set consists of 9087 , the validation set of 3029 , and the test set of 3029 images.

\subsection{EXPERIMENTAL RESULTS AND ANALYSIS}

In this paper, comparison experiments were conducted with different multi-feature fusion algorithms under the same data pre-processing, and the experimental results are shown in Table 1.

Table 1 Comparison of classification accuracy of multi-feature fusion algorithms

\begin{tabular}{rllll}
\hline & AlexNet & VGG16 & ResNet & DenseNet \\
\hline Original Algorithm & $91.2 \%$ & $92.0 \%$ & $88.5 \%$ & $88.7 \%$ \\
Algorithm in this paper & $92.6 \%$ & $93.1 \%$ & $91.2 \%$ & $91.0 \%$ \\
\hline
\end{tabular}

As shown in Table 1 above, the accuracy of the multi-feature fusion-based algorithm has improved significantly over the original algorithm with different deep learning networks by $1.6 \%, 1.1 \%, 2.7 \%$, and $2.3 \%$, respectively. Therefore, the multi-feature fusion-based rectal tumor recognition method proposed in this paper was proved to be effective through comparative experiments.

\section{CONCLUSION AND PROSPECT}

A rectal tumor is one of the most lethal malignant tumors in the world. It is of great importance to identify them accurately from CT images. In this paper, a multi-feature fusion-based convolutional neural network is proposed for rectal tumor recognition, combining traditional machine learning features and deep convolution features. By comparing the results of the experiments, the proposed multi-feature fusion-based convolutional neural network rectal tumor identification method is proved to obtain higher accuracy rates. For doctors, it can improve the diagnosis and identification of rectal tumors and reduce misdiagnosis and missed diagnosis, and for patients, it can provide valuable treatment time, which is of great significance for clinical diagnosis. This method is not only an important application of artificial intelligence technology in the field of medical imaging but also an important part of intelligent medicine.

As only three traditional image feature extraction methods were selected for study in this paper, they are computationally intensive. Further research will involve more image feature extraction methods for image classification and recognition to obtain higher accuracy and optimize the algorithm to reduce the computation time. Furthermore, the multi-feature fusion approach may be applied to other areas of computer vision, such as target detection and image segmentation.

\section{REFERENCES}

[1] Peng Junjie, Zhu Ji, Liu Fangqi, Chen Zhiyu, Tong Tong, Huang Dan, Li Wenhua, Yang Lifeng, Expert consensus on diagnosis and treatment of locally advanced rectal cancer in China [J], China Oncology, Vol. 27, No. 1, pp. 41-80, 2017 
[2] Amira A. Mahmoud, Walid El-Shafai, Taha E. Taha, El-Sayed M. El-Rabaie, Osama Zahran, Adel S. El-Fishawy, Fathi E. Abd El-Samie, A statistical framework for breast tumor classification from ultrasonic images [J],. Multimedia Tools and Applications, Vol. 80, 2020 (prepublish). https://doi.org/10.1007/s11042-020-08693-0

[3] Xiaofu Huang, Ming Chen, Peizhong Liu, Yongzhao Du, Lei Chen, Texture Feature-Based Classification on Transrectal Ultrasound Image for Prostatic Cancer Detection [J], Computational and Mathematical Methods in Medicine 2020, 2020.

https://doi.org/10.1155/2020/7359375

[4] Wadhah Ayadi, Imen Charfi, Wajdi Elhamzi, Mohamed Atri, Brain tumor classification based on hybrid approach [J], The Visual Computer, 2020 (prepublish).

https://doi.org/10.1007/s00371-020-02005-1

[5] S.L. Aarthy, S. Prabu, Classification of breast cancer based on thermal image using support vector machine [J], Int. J. of Bioinformatics Research and Applications, Vol. 15, No. 1, 2019. https://doi.org/10.1504/IJBRA.2019.10019395

[6] Khalid Usman, Kashif Rajpoot, Brain tumor classification from multi-modality MRI using wavelets and machine learning [J], Pattern Analysis and Applications, Vol. 20, No. 3, 2017. https://doi.org/10.1007/s10044-017-0597-8

[7] Yao Yao, Gou Shuiping, Tian $\mathrm{Ru}$, Zhang Xiangrong, He Shuixiang, Zhou Zhiguo. Automated Classification and Segmentation in Colorectal Images Based on Self-Paced Transfer Network [J], BioMed Research International, Vol. 2021, 2021.

https://doi.org/10.1155/2021/6683931

[8] H.G. Nguyen, A. Blank, H.E. Dawson, et al. Classification of colorectal tissue images from high throughput tissue microarrays by ensemble deep learning methods [J], Scientific Reports, Vol. 11, No. 2371, 2021. https://doi.org/10.1038/s41598-021-81352-y

[9] S. Poudel, Y.J. Kim, D.M. Vo, et al. Colorectal Disease Classification Using Efficiently Scaled Dilation in Convolutional Neural Network [J], IEEE Access, PP (99):1-1, 2020.

https://doi.org/10.1109/ACCESS.2020.2996770

[10] Natinai Jinsakul, Cheng-Fa Tsai, Chia-En Tsai, Pensee Wu, Enhancement of Deep Learning in Image Classification Performance Using Xception with the Swish Activation Function for Colorectal Polyp Preliminary Screening [J], Mathematics, Vol. 7, No. 12, 2019. https://doi.org/10.3390/math7121170

[11] Koziarski M, Two-Stage Resampling for Convolutional Neural Network Training in the Imbalanced Colorectal Cancer Image Classification [J], 2020.

[12] Navneet Dalal, Bill Triggs, Histograms of Oriented Gradients for Human Detection, International Conference on Computer Vision \& Pattern Recognition (CVPR '05), Jun 2005, San Diego, United States. pp. 886-893, 10.1109/CVPR.2005.177. inria-00548512.

[13] T. Ojala, M. Pietikäinen and D. Harwood, Performance evaluation of texture measures with classification based on Kullback discrimination of distributions, Proceedings of the $12^{\text {th }}$ IAPR International Conference on Pattern Recognition (ICPR 1994), 1, pp. 582-585, 1994. https://doi.org/10.1109/ICPR.1994.576366

[14] Ming-Kuei Hu, Visual pattern recognition by moment invariants, in IRE Transactions on Information Theory, Vol. 8, No. 2, pp. 179-187, February 1962. 
https://doi.org/10.1109/TIT.1962.1057692

[15] A. Krizhevsky, I. Sutskever, G. Hinton, ImageNet Classification with Deep Convolutional Neural Networks [C]// NIPS, Curran Associates Inc, 2012.

[16] K. Simonyan, A. Zisserman, Very Deep Convolutional Networks for Large-Scale Image Recognition [J], Computer Science, 2014.

[17] K. He, X. Zhang, S. Ren, et al. Deep Residual Learning for Image Recognition [J], 2016 IEEE Conference on Computer Vision and Pattern Recognition (CVPR), 2016. https://doi.org/10.1109/CVPR.2016.90

[18] G. Huang, Z. Liu, V.D.M Laurens, et al. Densely Connected Convolutional Networks [J], 2017 IEEE Conference on Computer Vision and Pattern Recognition (CVPR), 2017. https://doi.org/10.1109/CVPR.2017.243

[19] Zhao Jumin, Zhang Chen, Li Dengao, Niu Jing. Combining multi-scale feature fusion with multi-attribute grading, a CNN model for benign and malignant classification of pulmonary nodules. [J], Journal of digital imaging, Vol. 33, 2020.

https://doi.org/10.1007/s10278-020-00333-1

[20] Elene Firmeza Ohata, João Victor Souza das Chagas, Gabriel Maia Bezerra, Mohammad Mehedi Hassan, Victor Hugo Costa de Albuquerque, Pedro Pedrosa Rebouças Filho. A novel transfer learning approach for the classification of histological images of colorectal cancer [J], The Journal of Supercomputing, 2021 (prepublish).

[21] Young Joo Yang, Bum-Joo Cho, Myung-Je Lee, Ju Han Kim, Hyun Lim, Chang Seok Bang, Hae Min Jeong, Ji Taek Hong, Gwang Ho Baik, Automated Classification of Colorectal Neoplasms in White-Light Colonoscopy Images via Deep Learning [J], Journal of Clinical Medicine, Vol. 9, No. 5, 2020. https://doi.org/10.3390/jcm9051593

[22] M. Ali, S.O. Gilani, A. Waris, et al. Brain Tumour Image Segmentation Using Deep Networks [J], IEEE Access, Vol. 8, PP (99):1-1, 2020.

https://doi.org/10.1109/ACCESS.2020.3018160

[23] Y. Pei, X. Li, L. Mu, et al. Colorectal Tumor Segmentation Of CT Scans Based On A Convolutional Neural Network With An Attention Mechanism [J], IEEE Access, Vol. 8, PP (99):1-1, 2020.

[24] R. Ashraf, M.A. Habib, M. Akram, et al. Deep Convolution Neural Network for Big Data Medical Image Classification [J], IEEE Access, PP (99):1-1, 2020.

[25] S. Randhawa, A. Alsadoon, P. Prasad, et al. Deep learning for liver tumour classification: enhanced loss function [J], Multimedia Tools and Applications, Vol. 80, 2020.

https://doi.org/10.1007/s11042-020-09900-8

[26] Z. Chen, X. Wang, K. Yan, et al. Deep multi-scale feature fusion for pancreas segmentation from CT images [J], International Journal of Computer Assisted Radiology and Surgery, Vol. 15, No. 17, 2020. https://doi.org/10.1007/s11548-020-02117-y

[27] S. Wan, H.C. Lee, X. Huang, et al. Integrated local binary pattern texture features for classification of breast tissue imaged by optical coherence microscopy [J], Medical Image Analysis, Vol. 38, pp. 104-116, 2017. https://doi.org/10.1016/j.media.2017.03.002 
[28] V. Blanes-Vidal, G. Baatrup, E.S. Nadimi, Machine Learning-Based Colorectal Cancer Detection[C]// Association for Computing Machinery - Research in Adaptive and Convergent Systems (RACS 2018), pp. 43-46, 2018.

https://doi.org/10.1145/3264746.3264785

[29] R. Mostafiz, M.S. Uddin, N.A. Alam, et al. MRI-based brain tumor detection using the fusion of histogram oriented gradients and neural features [J], Evolutionary Intelligence, Vol. 14, 2021. https://doi.org/10.1007/s12065-020-00550-1

[30] J. Lee, J.E. Oh, M.J. Kim, et al. Multi-Task Learning with a Fully Convolutional Network for Rectum and Rectal Cancer Segmentation [J], 2019.

[31] W. Liu, M. Juhas, Y. Zhang, Fine-Grained Breast Cancer Classification with Bilinear Convolutional Neural Networks (BCNNs) [J], Frontiers in Genetics, 2020.

https://doi.org/10.3389/fgene.2020.547327 